
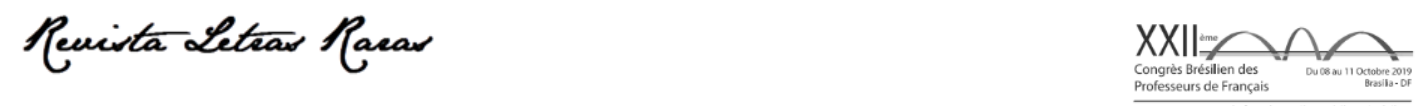

Actes du XXIlème Congrès brésilien des professeurs de français (dans Édition spéciale de la Revue Letras Raras 2020)

ISSN : 2317-2347 - v. 9, Dossier Spécial (2020)

Todo o conteúdo da RLR está licenciado sob Creative Commons Atribuição 4.0 Internacional

\title{
Quelques réflexions sur le traitement des difficultés de compréhension et/ou de traduction du français, avec trois exemples: « balade », « toit » et «parade» 1
}

Taise Soares Peixoto Nascimento*

Étudiante en lettres - portugais et français à l'Université Fédérale du Rio Grande do Sul - UFRGS (Porto Alegre, RS, Brésil). Boursière PIBIC-CNPq/UFRGS. L'auteure remercie le CNPq de la bourse.

https://orcid.org/0000-0003-3456-6785

\section{Robert Ponge*}

Professeur titulaire retraité de l'institut des lettres de l'UFRGS (Porto Alegre, RS, Brésil), professeur invité du centre d'études doctorales en lettres de la même université où il enseigne la littérature française et la traduction.

iD https://orcid.org/0000-0002-1078-821

Reçu le 20 oct. 2020. Approuvé le 04 nov. 2020.

\section{Comment citer cet article :}

NASCIMENTO, Taíse Soares Peixoto; PONGE, Robert. Quelques réflexions sur le traitement des difficultés de compréhension et/ou de traduction du français, avec trois exemples: « balade », " toit » et "parade». In: CONGRES BRESILIEN DES PROFESSEURS DE FRANÇAIS, 22., 2019, Brasilia. Actes du XXIIème Congrès Brésilien des Professeurs de Français. Édition spéciale de la Revue Letras Raras: Campina Grande. EDUFCG. nov. 2020, p. 368-384.

\section{RÉSUMÉ}

Ce travail présente quelques-unes des activités du projet que nous menons à l'UFRGS sur les difficultés de compréhension et/ou de traduction du français langue étrangère. II a deux objectifs : élaborer une classification des types de difficultés de compréhension et/ou de traduction du français ; et créer un glossaire contenant des mots, des expressions et des constructions qui provoquent des difficultés concrètes. Nous considérons comme des difficultés les problèmes, les obstacles, les incompréhensions ou les pièges qui apparaissent dans les processus de compréhension du français par les brésiliens et/ou de sa traduction en portugais du Brésil. Nous définissons comme des difficultés concrètes les occurrences de difficultés qui se manifestent dans le discours. Notre article examine les deux objectifs

\footnotetext{
${ }^{1}$ Cet article a son point de départ dans un travail qui va être publié en portugais par les auteurs dans: Anais do $2^{\circ}$ ENCONTRA (2020). Rio Grande, RS: Editora da FURG, sous presse. Quelques paragraphes en ont été traduits ou adaptés en français, mais le présent article s'en différencie substantiellement parce que les entrées étudiées sont différentes et qu'il a été considérablement augmenté.
}

taise.speixoto@gmail.com 

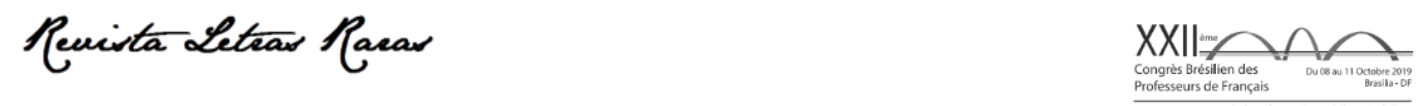

Actes du XXIlème Congrès brésilien des professeurs de français (dans Édition spéciale de la Revue Letras Raras 2020)

ISSN : 2317-2347 - v. 9, Dossier Spécial (2020)

Todo o conteúdo da RLR está licenciado sob Creative Commons Atribuição 4.0 Internacional

du projet et leurs rapports de complémentarité, nous montrons quatre types de difficultés que nous étudions et, à partir de trois exemples, le chemin suivi pour préparer des articles pour le glossaire.

MOTS-CLÉS: Français langue étrangère (FLE) ; Difficultés de compréhension ; Difficultés de traduction ; Françaisportugais.

\section{Introduction}

Ce travail vise à présenter le projet de recherche intitulé « Les difficultés de compréhension et/ou de traduction du français », mené à l'UFRGS, sous la direction de Robert Ponge, et à exposer une partie des activités que nous y réalisons. Le projet a pour but de développer deux outils utiles pour l'enseignement, l'apprentissage et la pratique de la compréhension et/ou de la traduction du français langue étrangère (FLE).

Dans le présent exposé, nous abordons certains des types de difficultés de compréhension et/ou de traduction sur lesquels nous travaillons. Nous commentons aussi le processus d'élaboration d'articles pour un glossaire réunissant des difficultés concrètes de compréhension e/ou de traduction du français. Nous offrons trois exemples d'articles, "balade », " toit » et "parade », en élucidant quelles sont les difficultés qui ont apparu et comment nous les avons traitées. Tout au long du texte, nous montrons la relation entre ces deux activités (qui sont les deux objectifs du projet).

Tout d'abord, nous parlons du projet de recherche.

\section{Objectifs, concepts et méthodologie}

Le projet de recherche a deux objectifs : d'une part, examiner les divers types de difficultés de compréhension du français langue étrangère et/ou de sa traduction en portugais brésilien et élaborer une classification de ceux-ci ; d'autre part, organiser un glossaire contenant des mots, des expressions et des constructions qui provoquent généralement des difficultés concrètes de compréhension et/ou de traduction du français en portugais.

Qu'entendons-nous par traduction? Nous la définissons comme « l'acte d'énoncer dans une autre langue (ou langue cible) ce qui a été énoncé dans une langue source, en conservant », ou plutôt en cherchant à conserver « les équivalences sémantiques et stylistiques » (DUBOIS et alii, 2002, p. 487). Les autres références théoriques ou bibliographiques sont fournies dans le corps du texte. 

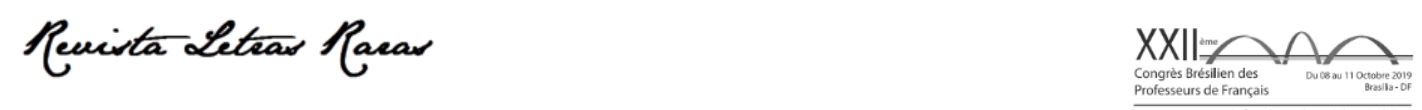

Actes du XXIlème Congrès brésilien des professeurs de français (dans Édition spéciale de la Revue Letras Raras 2020) ISSN : 2317-2347 - v. 9, Dossier Spécial (2020)

Todo o conteúdo da RLR está licenciado sob Creative Commons Atribuição 4.0 Internacional

Et ces difficultés, de quoi s'agit-il ? Nous considérons comme des difficultés les problèmes, les obstacles, les erreurs ou les pièges qui apparaissent d'ordinaire, chez les lusophones brésiliens, dans la compréhension du français et/ou dans sa traduction en portugais. Nous définissons comme des difficultés concrètes les occurrences de difficultés qui se manifestent dans le discours.

Dans la section suivante, nous nous penchons sur l'objectif théorique et expliquons comment nous l'étudions.

\section{L'objectif théorique : une classification des types de difficultés}

L'objectif théorique vise à analyser et à décrire les types de difficultés de compréhension et/ou de traduction du français langue étrangère et à en proposer une classification. Pour cela, nous avons d'abord utilisé, comme bases théoriques, des textes et des livres qui offrent des définitions de plusieurs types de difficultés et des façons différentes de les classer : Mounin (1963), Vinay et Darbelnet (1972), Rónai (1976a, 1976b), Portinho (1984), Rabadán (1991), Durieux (1999), entre autres. Ensuite, pour approfondir ces questions, nous lisons également des textes qui s'occupent spécifiquement de chaque type de difficulté, des moyens de les classer ou qui abordent des sujets voisins. En outre, chaque membre de notre équipe est encouragé à choisir un type spécifique de difficulté et à le traiter pendant son mémoire de licence, de master ou lors de communications dans des congrès.

Cela nous a permis de commencer à établir une classification des types de difficultés. Jusqu'à présent, nos efforts nous ont menés à en identifier plusieurs : les faux amis, les homonymes, les paronymes, la polysémie, les synonymes, les sens figurés, le calque, les abréviations et les sigles, les expressions idiomatiques, entre autres.

Cette typologie est utile pour savoir quelles sont les causes d'erreurs ou de difficultés dans la compréhension du FLE et dans sa traduction, et pour savoir les reconnaître dans leur apprentissage, elle est donc utile dans l'enseignement de ces deux capacités. Elle nous sert également à perfectionner la microstructure, la qualité, la précision et l'utilité des articles que nous préparons pour le glossaire dont nous allons parler d'ici peu.

Dans ce travail, nous allons montrer trois exemples d'articles produits pour le glossaire. L'entrée de chacun est un mot où habituellement se manifestent (ou peuvent se manifester) les 

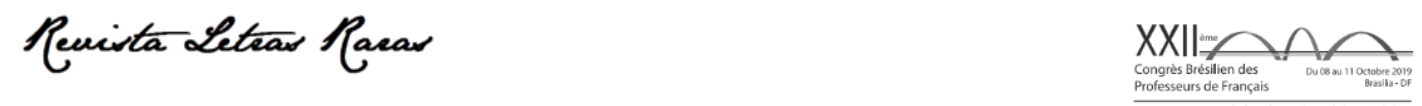

Actes du XXIlème Congrès brésilien des professeurs de français (dans Édition spéciale de la Revue Letras Raras 2020) ISSN : 2317-2347 - v. 9, Dossier Spécial (2020)

Todo o conteúdo da RLR está licenciado sob Creative Commons Atribuição 4.0 Internacional

types de difficultés de compréhension et/ou de traduction suivants : les faux amis, la polysémie, l'homonymie et les expressions (ou locutions) idiomatiques, types qu'il est maintenant nécessaire de définir.

De toutes les sortes de difficultés, celle des faux amis est la plus connue. Elle correspond à deux mots de langues différentes dont la forme, l'aspect est très semblable, cependant le sens de chacun est complètement différent (Rónai, 1976A, p.19). Cette ressemblance trompeuse conduit souvent le lecteur à un malentendu (et à une mauvaise traduction quand traduction il y a). Par exemple le mot français « pipe » peut être confondu avec le mot portugais pipa (cerf-volant) par sa ressemblance graphique et phonétique.

Moins connu, mais phénomène linguistique des plus courants, la polysémie se produit quand une unité linguistique, un mot a plusieurs sens (DUBOIS, 2012, p.369). Pourquoi peut-elle être un obstacle à la compréhension en langue étrangère ? D'abord, parce que la polysémie est ou peut être source d'ambiguïté dans tout milieu endolingue, c'est-à-dire un milieu où une même langue est commune aux interlocuteurs (DUBOIS, 2012, p.31, 370). Mais aussi, en ce qui concerne le FLE, parce qu'il est normal et courant qu'il y ait de nombreux vides, des angles morts, des trous, des crevasses sémantiques dans le vocabulaire actif et disponible des locuteurs allophones (en situation de langue étrangère). Par exemple, qu'ils ignorent qu'une matinée théâtrale, musicale ou dansante n'aura pas lieu le matin, mais pendant l'après-midi (ROBERT, 2017, p.446).

Polysémie : un seul mot, plusieurs sens. Par contre, il y a homonymie quand « on a plusieurs mots de sens distincts ayant la même orthographe, la même prononciation ou les deux en même temps » (CUNHA ; PONGE, 2010, p. 2), par exemple (1) desservir et (2) desservir. ॥ s'agit de deux verbes différents, mais de sens contraires. Le premier verbe informe qu'un moyen de transport s'arrête dans un lieu (ex. aucun train ne dessert ce village), le deuxième signifie débarrasser une table ou nuire à quelqu'un ou faire obstacle à l'exécution de quelque chose (ROBERT, 2017, p. 201).

Les deux cas (polysémie et homonymie) sont distincts, mais le problème, le danger est le même: la similitude (phonétique, graphique ou les deux) des homonymes crée un risque d'ambiguïté, de confusion, d'incompréhension, voire de contresens. Car enfin, de quel mot s'agitil ? Un autre problème causé par les homonymes peut se produire lors de la consultation d'un dictionnaire, « car l'usager peut ne pas s'apercevoir de l'occurrence de plusieurs articles ayant la 

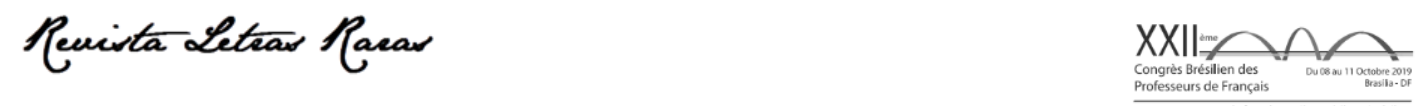

Actes du XXIlème Congrès brésilien des professeurs de français (dans Édition spéciale de la Revue Letras Raras 2020) ISSN : 2317-2347 - v. 9, Dossier Spécial (2020)

Todo o conteúdo da RLR está licenciado sob Creative Commons Atribuição 4.0 Internacional

même orthographe, ce qui peut avoir comme conséquence des erreurs de compréhension et de traduction »(CUNHA; PONGE, 2011, p. 5).

Enfin, une expression ou locution idiomatique est un groupe de mots qui « fonctionne comme un ensemble, une unité », c'est-à-dire que " son sens est global, non déductible de la somme du sens des mots qui la compose » (SILVA ; PONGE, 2017, p.2). Par exemple la locution "avoir avalé un pépin" (manière populaire de dire qu'une femme est enceinte) est opaque pour quelqu'un qui la rencontre pour la première fois parce que son sens global est très différent de la somme de ses composants. C'est pourquoi une locution idiomatique constitue fréquemment une difficulté de compréhension, et aussi une difficulté de traduction, car il est nécessaire de trouver dans la langue cible un syntagme qui « conserve les équivalences sémantiques, [...] stylistiques, expressives [...] de la langue source et dont le caractère idiomatique repose sur une image [...] qui, de préférence, soit identique à celle de la langue source [...] ou qui possède avec celle-ci un certain rapport [...]» (SILVA ; PONGE, 2017, p.2).

Comment mettons-nous à profit nos connaissances relatives à la classification des types de difficultés pour améliorer la préparation des articles du glossaire que nous organisons ? C'est ce que nous allons voir dans la partie suivante.

\section{L'objectif pratique : un glossaire de difficultés concrètes}

Cet objectif cherche à organiser un glossaire contenant des articles relatifs à des mots, expressions ou constructions qui représentent des difficultés concrètes de compréhension et/ou de traduction. Comme bibliographie initiale, nous utilisons trois petits guides ou dictionnaires brésiliens de difficultés concrètes de compréhension et/ou de traduction du français : Rónai, 1983 ; Xatara e Oliveira, 2008 ; Bath e Biato, 1998. Nous faisons un collationnement critique de ces trois ouvrages, et mettons à profit les leçons que nous en tirons pour améliorer la microstructure des articles que nous préparons pour le glossaire.

Le choix d'un mot pour qu'il devienne entrée de notre glossaire est, dans un premier temps, motivé par son occurrence dans l'un des trois guides ou dictionnaires que nous prenons comme bases de données initiales. Mais d'autres mots, expressions ou constructions peuvent également devenir articles du glossaire, même s'ils ne figurent pas dans ces ouvrages. La nomenclature du glossaire est en effet alimentée également par des mots, des syntagmes, des structures qui ont 

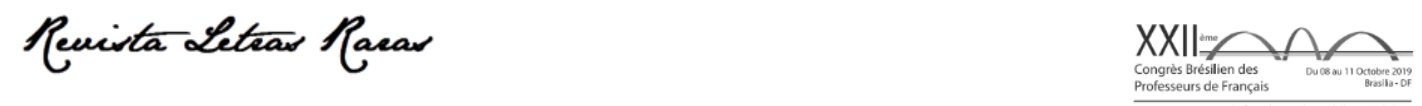

Actes du XXIlème Congrès brésilien des professeurs de français (dans Édition spéciale de la Revue Letras Raras 2020) ISSN : 2317-2347 - v. 9, Dossier Spécial (2020)

Todo o conteúdo da RLR está licenciado sob Creative Commons Atribuição 4.0 Internacional

été identifiés comme des difficultés de compréhension et/ou de traduction et qui sont fournis par les membres de notre équipe de recherche, par nos étudiants, par nos collègues et qui proviennent de leur pratique de la langue française. Après avoir choisi le mot qui deviendra un article, nous commençons le processus de collationnement (que nous allons détailler ci-dessous).

Lors de la préparation des articles, nous faisons usage de dictionnaires unilingues de la langue française (Larousse, Petit Robert, huitième et neuvième édition du Dictionnaire de l'Académie française, TLFI) et de la langue portugaise (Aurélio, Borba, Caldas Aulete, Houaiss, Michaelis). Les dictionnaires de français permettent d'examiner les divers sens du mot ou de l'expression en question, ainsi que les définitions données par chaque dictionnaire. Par ailleurs, les dictionnaires de portugais servent à chercher des équivalents (des correspondants), à appréhender et à délimiter le contenu sémantique des équivalents, ainsi qu'à formuler la définition de chaque sens ; ce sont aussi des instruments utiles quand il s'agit de traduire le mot en question et les phrases citées en exemple. Dans le processus de rédaction des articles, il peut être nécessaire de se servir de dictionnaires bilingues ou semi-bilingues, mais ils sont maniés avec grande précaution, en gardant à l'esprit que ce sont des outils problématiques dont la fiabilité est réduite et la précision faible.

Maintenant, nous nous occupons du processus d'élaboration des articles pour le glossaire. soit essentiellement l'activité de collationnement et l'organisation interne des articles. À des fins didactiques, nous le faisons en illustrant nos explications avec des extraits des articles rédigés pour trois entrées : « balade », « toit » et « parade ».

\section{L'élaboration d'articles pour le glossaire : le collationnement}

Pour la production d'un article pour le glossaire, nous commençons par son collationnement critique dans chacun des trois guides ou dictionnaires mentionnés (RÓNAI, 1983; XATARA e OLIVEIRA, 2008; BATH e BIATO, 1998), c'est-à-dire que, pour une même entrée, nous prenons l'article correspondant dans chacun des trois et que nous comparons leur forme, leur contenu, la(les) définition(s) donnée(s), les exemples, les équivalents, etc.

Pour illustrer le processus de collationnement, nous avons choisi une entrée (« balade »), qui figure dans deux des trois ouvrages de référence. Comment procédons-nous ? Nous organisons un tableau avec cinq colonnes (Fig. 1). Dans la première, nous avons l'entrée, et, dans 

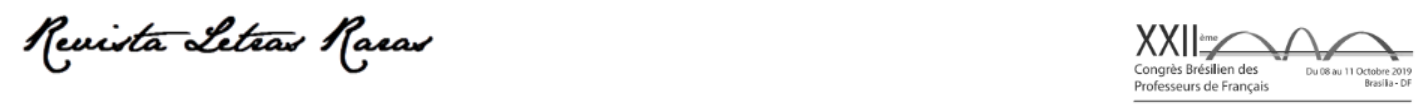

Actes du XXIlème Congrès brésilien des professeurs de français (dans Édition spéciale de la Revue Letras Raras 2020)

ISSN : 2317-2347 - v. 9, Dossier Spécial (2020)

Todo o conteúdo da RLR está licenciado sob Creative Commons Atribuição 4.0 Internacional

les trois colonnes suivantes, nous avons l'article comme il apparaît dans chacun des trois lexiques utilisés. De cette façon, nous avons une organisation visuelle qui met côte à côte les articles d'une même entrée dans les trois ouvrages cités.

Figure 1 : Le processus de collationnement - article « balade »

\begin{tabular}{|l|l|l|l|l|}
\hline \hline Entrada & Rónai & $\begin{array}{l}\text { Xatara e } \\
\text { Oliveira }\end{array}$ & $\begin{array}{l}\text { Bath e } \\
\text { Biato }\end{array}$ & Verbete provisório \\
\hline Balade & $\begin{array}{l}\text { s.f. (pop.) } \\
\text { passeio }\end{array}$ & $\begin{array}{l}\text { f. Passeio: } \\
\text { L'été prochain } \\
\text { nous ferons } \\
\text { une balade par } \\
\text { la Bourgogne. } \\
\text { [balada: } \\
\text { ballade]. }\end{array}$ & & balade [ba.lad] s.f. \\
& & & \\
\hline
\end{tabular}

Que faisons-nous pendant le collationnement? Nous nous appliquons à observer si les difficultés que ce mot, expression ou construction provoque sont présentées clairement et sans risque d'ambiguïté ou d'erreur dans chacun des trois ouvrages de référence, et comment, dans notre glossaire, nous pouvons améliorer la structure des articles, la formulation des définitions et des équivalents ainsi que les exemples. Ce collationnement critique conduit à l'élaboration d'un article entièrement nouveau, article dit provisoire, provisório en portugais (voir plus bas).

La cinquième et dernière colonne contient le nouvel article, qui est travaillé comme suit.

\section{L'élaboration d'articles pour le glossaire : la microstructure des articles}

Comment les articles du glossaire sont-ils organisés, quelle est leur microstructure? Utilisons comme exemple le mot « toit». II est composé, d'abord, par l'entrée, suivie de sa transcription phonétique entre crochets et des informations sur sa classe grammaticale et sur son genre (Fig. 2).

Ensuite, il y a un ou plusieurs avertissement(s) pour indiquer le ou les type(s) de difficulté que le mot en question est susceptible de causer aux lusophones brésiliens. C'est ici que notre connaissance de la classification des types de difficultés se montre particulièrement utile, même si 


\section{Reuista Letear Pacar}

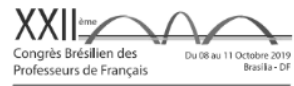

Actes du XXIlème Congrès brésilien des professeurs de français (dans Édition spéciale de la Revue Letras Raras 2020)

ISSN : 2317-2347 - v. 9, Dossier Spécial (2020)

Todo o conteúdo da RLR está licenciado sob Creative Commons Atribuição 4.0 Internacional

cela ne transparaît pas explicitement en conséquence du fait que nous évitons l'emploi du métalangage (voir ci-dessous).

Figure 2 : Organisation des articles - article « toit »

Verbete provisório

toit [twa] s.m.

$\triangle$ Não confundir com "toi".

$\triangle$ Falso amigo: o sentido literal não refere ao "teto" designando a parte interna superior de uma casa ou aposento.

$\triangle$ Não confundir com "toiture" (uso técnico).

Comme stratégie de visualisation, nous employons le symbole $₫$ avant chacun des avertissements. Nous cherchons à les formuler en langage aussi simple et clair que possible, sans recourir à des termes techniques de linguistique. Nous souhaitons ainsi faciliter la compréhension de tous ceux qui vont consulter le glossaire, pour qu'ils appréhendent directement, de façon pratique en quoi consiste la ou les difficulté(s), le ou les danger(s) caché(s) dans le mot, l'expression ou la tournure en question et dont les avertissements ont pour fonction de les prévenir.

Après les avertissements, comme les mots sont presque tous polysémiques nous fournissons les différents sens que le mot présente, en numérotant chacun. Au début de chaque sens sont apportés, entre parenthèses et en abrégé, des informations sur ses caractéristiques d'emploi, de niveau de langue, etc. Pour chaque sens, nous avons une définition, suivie d'une ou de plusieurs suggestion(s) d'équivalent (Fig. 3).

Figure 3 : Les sens - article « toit »

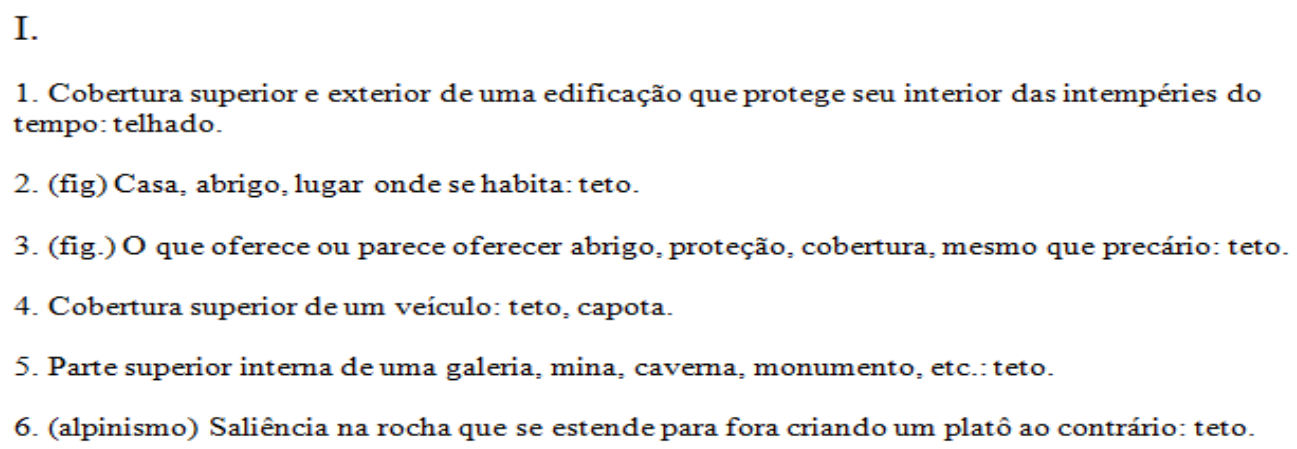



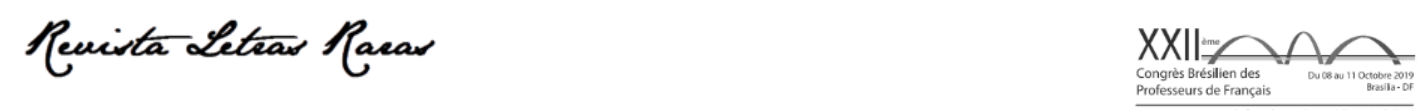

Actes du XXIlème Congrès brésilien des professeurs de français (dans Édition spéciale de la Revue Letras Raras 2020)

ISSN : 2317-2347 - v. 9, Dossier Spécial (2020)

Todo o conteúdo da RLR está licenciado sob Creative Commons Atribuição 4.0 Internacional

Dans la mesure du possible, nous donnons un ou plusieurs exemples adéquats et en contexte pour chaque sens numéroté. Pour chaque exemple, nous recherchons l'utilisation du mot, de l'expression ou de la construction en question dans des œuvres littéraires ou dans d'autres documents authentiques de pays francophones. De plus, nous incluons la source de l'exemple afin que, si nécessaire, son contexte puisse être consulté et sa source vérifiée. Nous fournissons également une traduction de la phrase en portugais du Brésil (Fig.4).

Figure 4 : Les exemples - article « toit »

1. Cobertura superior e exterior de uma edificação que protege seu interior das intempéries do tempo: telhado.

Le toit est soutenu par une charpente qui porte sur quatre gros piliers en bois.

(STENDHAL. Le Rouge et le Noir. Paris: Le Divan, 1927) "O telhado é sustentado por uma armação apoiada sobre quatro grossos pilares de madeira."

À la fin de l'article, viennent les locutions où se trouve le mot en question (Fig. 5). Chacune est suivie d'un ou de plusieurs équivalent(s) ainsi que d'au moins un exemple authentique de son emploi, accompagné de sa traduction en portugais.

Figure 5 : Les locutions - article « toit» 

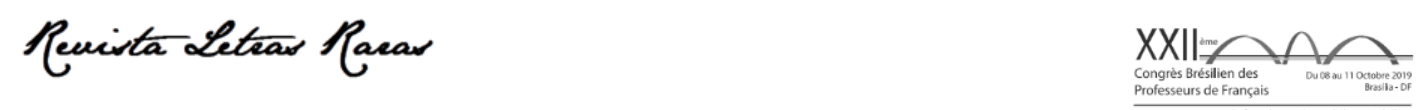

Actes du XXIlème Congrès brésilien des professeurs de français (dans Édition spéciale de la Revue Letras Raras 2020)

ISSN : 2317-2347 - v. 9, Dossier Spécial (2020)

Todo o conteúdo da RLR está licenciado sob Creative Commons Atribuição 4.0 Internacional

II. Loc.

Sous le même toit: sob o mesmo teto.

Madame de Rênal eut un sang-froid inaltérable pendant toute cette pénible conversation, de laquelle dépendait la possibilité de vivre encore sous le même toit avec Julien. (STENDHAL. Le Rouge et le Noir. Paris: Le Divan, 1927)

"Madame de Rênal manteve um sangue frio inalterável durante toda essa penosa conversa, da qual dependia a possibilidade de viver ainda sob o mesmo teto que Julien."

Loger/habiter/vivre sous le toit : morar em um quarto no último piso de uma casa ou prédio, logo abaixo do telhado (em geral, trata-se de um quarto alugado, sem conforto, até precário): morar sob o telhado, no sótão.

L'inconvénient majeur de mon appartement c'est le fait qu'il soit

TRIANGULAIRE. Vous allez rire mais du haut de mon $1 \mathrm{~m} 72$, je ne me suis jamais autant cognée la tête qu'en habitant sous les toits et qu'en allant aux toilettes [oui c'est ridicule]. (https://bit.ly/3alRW6x) "O maior inconveniente do meu apartamento é o fato de ele ser TRIANGULAR. Vocês vão rir mas, do alto dos meus $1,72 \mathrm{~m}$, eu nunca bati tanto a cabeça como quando morei num sótão e ia ao banheiro [sim, é ridículo]."

(fig) Le toit paternel: a casa patema, teto paterno.

Son fils, Pierre, en grande difficultéfinancière vient, lui aussi, chercher asile sous le toit paternel.(https//bit.ly/2FmR4Di) "Seu filho, Pierre, em grande dificuldade financeira, vem, ele também, procurar asilo sob o teto paterno."

Les articles sont alors considérés comme provisoires, parce que leur processus d'élaboration englobe des révisions avec les autres membres de notre équipe de recherche, discussions où il est vérifié : si les avertissements initiaux sont clairs et s'ils sont suffisants ; si tous les sens du mot ont été explorés ; si les définitions élaborées sont bonnes ; si les suggestions d'équivalents de chaque sens sont adéquates ; si l'exemple proposé correspond au sens qu'il prétend expliciter, s'il est approprié au contexte situationnel d'utilisation d'un glossaire ; etc.

Maintenant, nous expliquons en détail l'article « toit ».

7 Exemple d'article : « toit » et les avertissements en début d'article

Le mot « toit » ne figurait dans aucun des trois ouvrages cités. Nous avons décidé de l'ajouter au répertoire du glossaire pendant une traduction, lorsque nous avons identifié qu'il suscitait des difficultés de compréhension et de traduction.

Quelles difficultés ? Certaines sont inscrites (mais sans métalangage) dans les trois avertissements situés au début de l'article (Fig. 6). Nous en explicitons maintenant le métalangage 

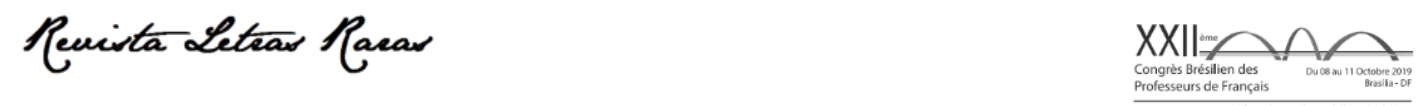

Actes du XXIlème Congrès brésilien des professeurs de français (dans Édition spéciale de la Revue Letras Raras 2020) ISSN : 2317-2347 - v. 9, Dossier Spécial (2020)

Todo o conteúdo da RLR está licenciado sob Creative Commons Atribuição 4.0 Internacional

sous-jacent. Le premier avertissement alerte que l'homophonie (forme phonique de l'homonymie) peut constituer une difficulté de compréhension, parce que l'on peut confondre le mot « toit » avec le pronom « toi », à cause de leur prononciation identique [twa].

Le deuxième indique un danger, celui du faux ami : confondre « toit » et le mot portugais teto, soit une difficulté de compréhension. Pourquoi ? Parce que l'orthographe des deux mots a quelques ressemblances, parce que les deux termes appartiennent au vocabulaire de l'architecture et que le sens premier des deux désigne un type de couverture supérieure, ce qui incite à considérer « toit » comme équivalent de teto, sauf que la couverture n'est pas la même : dans leur sens premier, « toit » signifie telhado et teto veut dire « plafond », risque de contresens.

Figure 6 : Article « toit » - les avertissements

toit [twa] s.m.

$\triangle$ Não confundir com "toi".

$\triangle$ Falso amigo: o sentido literal não refere ao "teto" designando a parte interna superior de uma casa ou aposento.

$\triangle$ Não confundir com "toiture" (uso técnico).

Le troisième avertissement signale une difficulté de compréhension parce qu'il renvoie à deux traits distinctifs qu'un allophone a de la difficulté à percevoir : a) « toiture » appartient au vocabulaire technique, et b) désigne le toit + l'armature du toit. L'avertissement signale aussi une difficulté de traduction parce que les mots « toit » et " toiture " se traduisent tous deux, dans certains cas, par le même mot en portugais, telhado, mais pas toujours.

D'autres difficultés, qui ne sont pas inscrites dans les avertissements, sont traitées dans le corps de l'article « toit »; elles découlent de la polysémie, des emplois figurés et des emplois idiomatiques. Nous les abordons dans l'article suivant.

\section{Autre exemple d'article : « parade »}

L'entrée «parade » figure dans un seul des ouvrages de référence (Xatara et Oliveira, 2008, p.98). Entièrement nouveau, l'article que nous avons élaboré commence par deux avertissements, chacun correspondant à une difficulté de compréhension (Fig. 7). 

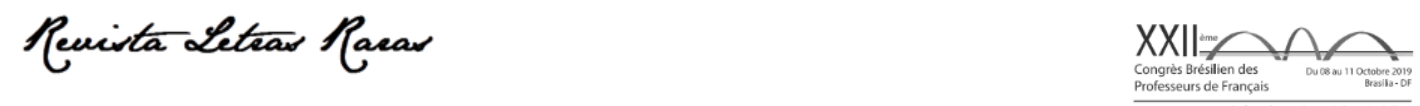

Actes du XXIlème Congrès brésilien des professeurs de français (dans Édition spéciale de la Revue Letras Raras 2020) ISSN : 2317-2347 - v. 9, Dossier Spécial (2020)

Todo o conteúdo da RLR está licenciado sob Creative Commons Atribuição 4.0 Internacional

Le premier avertissement se rapporte à l'homonymie, parce qu'il y a trois mots « parade » différents. Chacun a son sémantisme propre, distinct des deux autres, mais les trois sont de forme identique, d'où le risque qu'il y ait difficulté de compréhension. Comment différencier les trois noms dans notre glossaire ? En plus de l'avertissement initial, nous utilisons le symbole d'un nombre inscrit dans un cercle (1) avant chaque entrée : (1) Parade, (2) Parade et (3) Parade, pour marquer que ce sont trois mots différents.

Le deuxième avertissement alerte le lecteur qu'un danger menace : le faux ami, qui représente également une difficulté de compréhension. Pourquoi ? Parce que la ressemblance graphique entre le mot français " parade » et le mot portugais parada suggère une identité sémantique qui se confirme parfois (dans le sens de défilé, aussi en escrime et en équitation), parfois mais pas toujours ! Parce que le mot français, différemment de son sosie portugais, ne réfère jamais à une pause (un temps d'arrêt) ni à un lieu d'arrêt (arrêt d'autobus, station de métro, etc.). D'autre part, parce qu'il peut, entre autres, signifier également « ostentation, affectation », sens que n'a jamais le mot portugais.

Figure 7 : Article « parade » - les avertissements

\section{parade [parad] s.f.}

$\triangle$ Não confundir (1) parade, (2) parade e (3) parade.

$\triangle$ Falso amigo: não significa "parada" no sentido de pausa, ação ou resultado de parar, salvo em (3) parade. Também não significa "parada" no sentido de ponto de parada de transportes coletivos.

De même que pour « toit », d'autres types de difficultés (qui ne figurent pas dans les avertissements) sont traités dans le corps de l'article « parade ». Difficultés de compréhension (et parfois de traduction), ce sont la polysémie (donc les différents emplois et sens découlant de celleci) et les expressions idiomatiques. Nous allons en montrer quelques-uns. Rappelons que, pour « parade ", nous avons trois homonymes, trois mots différents (donc trois entrées distinctes : (1) Parade, (2) Parade et (3) Parade) et que nous numérotons les sens découlant de la polysémie de chaque mot (Fig. 8).

Figure 8 : Article « parade » - les sens 


\section{Reuista Letras Racar}

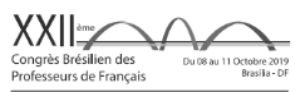

Actes du XXIlème Congrès brésilien des professeurs de français (dans Édition spéciale de la Revue Letras Raras 2020)

ISSN : 2317-2347 - v. 9, Dossier Spécial (2020)

Todo o conteúdo da RLR está licenciado sob Creative Commons Atribuição 4.0 Internacional

\section{(1) parade s.f.}

1. Exibição, ostentação que se faz de alguma coisa ou de alguma qualidade: ostentação, exibição.

2. (Mil.) Desfile de tropas em datas comemorativas, revista de tropas: parada, desfile.

3. Desfile civil em dias festivos: parada.

4. Apresentação, exibição feita no exterior de teatro, circo ou evento para chamar a atenção do público: parada, desfile.

5. (biologia) Comportamento de alguns animais quando, normalmente, o macho se aproxima da fêmea em busca do acasalamento: cortejo (nupcial, sexual), exibição.

\section{(2) parade s.f.}

1. (Esgrima) Ato de se defender de um golpe: parada, bloqueio.

2. (Fig.) Ação de defesa, resposta a uma situação, afirmação ou acusação: riposta, réplica, resposta, revide, solução, saída.

\section{(3) parade s.f.}

1. (Equitação) Ação de parar um cavalo: parada.

Chaque élément numéroté fournit une définition du mot, une ou plusieurs suggestion(s) d'équivalent en portugais, un exemple d'utilisation du mot en contexte, dans une phrase authentique et une traduction en portugais de celle-ci (Fig. 9).

Figure 9 : Article « parade » - les exemples 

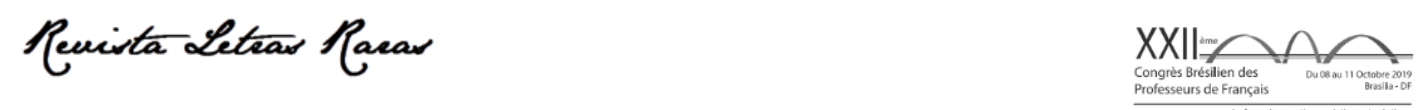

Actes du XXIlème Congrès brésilien des professeurs de français (dans Édition spéciale de la Revue Letras Raras 2020)

ISSN : 2317-2347 - v. 9, Dossier Spécial (2020)

Todo o conteúdo da RLR está licenciado sob Creative Commons Atribuição 4.0 Internacional

(1) parade s.f.

1. Exibição, ostentação que se faz de alguma coisa ou de alguma qualidade: ostentação, exibição.

Plumes et poils en parade au salon avicole de Charleville-Mézières.

(https://bit.ly/2E01nfM) "Penas e pelos em exibição na exposição avícola de

Charleville-Mézières."

$N^{\prime} y$ a-t-il pas dans ces affiches excessives d'abnégation et d'honneur beaucoup d'ostentation? C'est plutôt parade qu'autre chose. (Grand Robert - Hugo, l'Homme qui rit, II, i, i, ii) "Será que não há, nessas mostras excessivas de abnegação e de honra, muita ostentação? É mais exibição do que outra coisa."

Enfin, le cas échéant, il y a une section consacrée aux expressions idiomatiques. Chacune reçoit une ou plusieurs suggestion(s) d'équivalent, un exemple d'utilisation de la locution en contexte, dans une phrase authentique et une traduction en portugais de celle-ci (Fig. 10).

Figure 10 : Article « parade » - parade 1, quelques locutions

(1) parade s.f.

II. Loc.

De parade: de exibição, de ostentação, de desfile, de cerimônia, de festa, de gala.

C'était une belle arme (une épée), riche sans ornementation superflue, une arme de combat et non de parade. (GAUTIER, T. le Capitaine Fracasse, ix.) "Era uma bela arma (uma espada), rica, sem ormamentação supérflua, uma arma de combate e não de exibição."

Oh! Le manteau de parade! (GENET, Jean. Les bonnes, 1947) "Ah! O mantô de festa!"

Lit de parade: cama mortuária, leito mortuário.

Comme tous les lits de parade, ce lit n'avait pas de draps, les deux médecins déposèrent madame Graslin sur le couvre-pied [...]. (Balzac. Le Curé de village, P1., t. VIII, p. 753.)

"Como todo leito mortuário, esse leito não tinha lençóis, os dois médicos depuseram madame Graslin sobre a colcha [...]."

Cheval de parade: cavalo de desfile, palafrém.

Le service du festin fut commandé par les plus hauts barons du royaume [...], montés sur leurs chevanx de parade et vêtus de drap d'or. (TLFi) "O serviço do festim foi regido pelos mais altos barões do reino [...], montados em seus palafréns e vestidos com tecidos de ouro." 

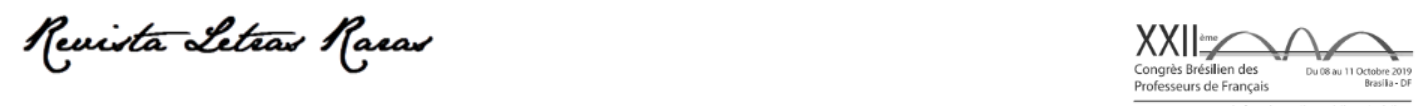

Actes du XXIlème Congrès brésilien des professeurs de français (dans Édition spéciale de la Revue Letras Raras 2020)

ISSN : 2317-2347 - v. 9, Dossier Spécial (2020)

Todo o conteúdo da RLR está licenciado sob Creative Commons Atribuição 4.0 Internacional

Comme on a pu le voir, nous faisons un grand effort pour que les avertissements et la microstructure (l'organisation) des articles contribuent à informer les lusophones brésiliens des diverses difficultés, des dangers que le mot en question peut causer.

\section{Quelques considérations finales}

Comme il a été possible de l'observer au long de ce travail, notre projet de recherche intervient sur deux terrains. D'une part, l'objectif théorico-descriptif prétend élaborer une classification des types de difficulté de compréhension et/ou de traduction qui aide les étudiants, les enseignants et les traducteurs du FLE à comprendre les problèmes divers auxquels ils font face dans leur pratique quotidienne de ces deux aptitudes (ou habiletés).

D'autre part, l'objectif pratique veut organiser un glossaire des difficultés concrètes de compréhension et/ou de traduction, lexique dont la nomenclature reprend celle de trois petits dictionnaires brésiliens de difficultés, mais enrichie des apports dûs aux suggestions de collègues et des membres de notre équipe de recherche. En outre, la microstructure des articles est très différente de celle des trois ouvrages cités.

Nous avons expliqué comment nous menons la réflexion théorique sur les phénomènes linguistiques et extralinguistiques qui engendrent des difficultés concrètes de compréhension et/ou de traduction du FLE pour les lusophones brésiliens. Nous avons également montré comment elle inspire la microstructure, les caractéristiques et la rédaction des articles du glossaire.

Si l'on nous demande quelle est l'idée ou la motivation centrale qui parcourt tout le projet, nous répondons que c'est la finalité didactique : élaborer deux outils qui soient utiles et didactiquement efficaces pour l'enseignement, l'apprentissage et la pratique de la compréhension du FLE et de sa traduction en portugais du Brésil. Nous espérons que toute la démarche du présent article en est la preuve. 

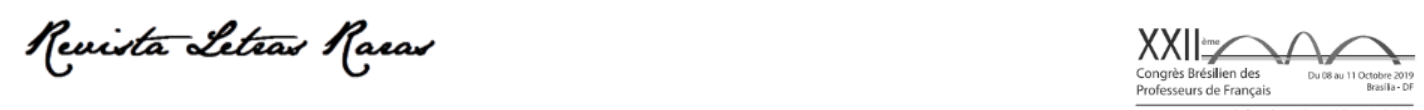

Actes du XXIlème Congrès brésilien des professeurs de français (dans Édition spéciale de la Revue Letras Raras 2020) ISSN : 2317-2347 - v. 9, Dossier Spécial (2020)

Todo o conteúdo da RLR está licenciado sob Creative Commons Atribuição 4.0 Internacional

\section{Références}

BATH, S.; BIATO, O. Les faux amis e outras peculiaridades da língua francesa. Brasília : Ed. UnB, 1998.

BORBA, F. S. Dicionário UNESP do português contemporâneo. São Paulo : Editora UNESP, 2004.

CUNHA, D. A. B.; PONGE, R. Les mots polysémiques et homonymiques dans un dictionnaire des difficultés de compréhension et traduction du FLE: quelques possibilités de présentation des articles. In: XV SEDIFRALE 2010 - 15ème Congrès latino-américain de professeurs de français, 15, 2010, Rosario. Rosario (Argentina): Clé international/FIPF/FAPF, 2010. p. 1-5. CléUSB/Pendrive.

CUNHA, D. A. B.; PONGE, R. Le traitement des mots polysémiques et celui des mots homonymiques dans un glossaire des difficultés de compréhension et de traduction du FLE. In: XVIIle Congrès Brésilien des Professeurs de Français, 18, 2011, Curitiba. (Apresentação de Trabalho/Comunicação) (Inédito, comunicado pelos autores).

DAF/8 - Dictionnaire de l'Académie française, 8ème édition. Paris: Académie française, 1935. Versão online. Disponível em: [https://www.dictionnaire-academie.fr/]. Acesso em: 30 jun. 2020.

$\mathrm{DAF} / 9$ - Dictionnaire de l'Académie française, 9ème édition. Paris: Académie française, 1992 - ... Versão online. Disponível em: [https://www.dictionnaire-academie.fr/]. Acesso em: 30 jun. 2020.

DICIONÁRIO BRASILEIRO DA LÍNGUA PORTUGUESA MICHAELIS. São Paulo: Melhoramentos, 2018. Disponível em: [https://michaelis.uol.com.br/moderno-portugues/]. Acesso em: 30 jun. 2020.

DICIONÁRIO CALDAS AULETE. São Paulo: Lexikon, 2018. Disponível em: [http://www.aulete.com.br/]. Acesso em: 30 jun. 2020.

DUBOIS, J. et alii. Dictionnaire de linguistique et des sciences du langage. Paris: Larousse, 2002.

DURIEUX, C. La difficulté en traduction. Revue des lettres et de traduction. Université du SaintEsprit de Kaslik, (Liban), $n^{0} \quad 5$, 1999. Disponível em: [http://documents.irevues.inist.fr/bitstream/handle/2042/41863/1999_5_31-34.pdf?sequence=3]. Acesso em: 30 jun. 2020.

FERREIRA, A. B. de H. Novo dicionário Aurélio da língua portuguesa. Curitiba: Positivo, 2009.

HOUAISS, A. Dicionário da língua portuguesa. Rio de Janeiro: Objetiva, 2001.

LAROUSSE DICTIONNAIRE DE FRANÇAIS. Versão Online. Paris: Larousse, 2018. Disponível em: [http://www.larousse.fr/dictionnaires/francais/]. Acesso em: 30 jun. 2020.

MOUNIN, G. Les problèmes théoriques de la traduction. Paris: Editions Gallimard, 1963. (Coll. "Bibliothèque des Idées").

PORTINHO, W. M. (org.). A tradução técnica e seus problemas. São Paulo: Álamo, 1984.

RABADÁN, R. La inequivalencia: imposibilidad o límites? In: Equivalencia y traducción: problemática de la equivalencia translémica inglés-español. Léon: Universidad, Secretariado de Publicaciones, 1991, p. 109-173.

ROBERT, P. Le Robert poche. Paris: Le Robert, 2017. 

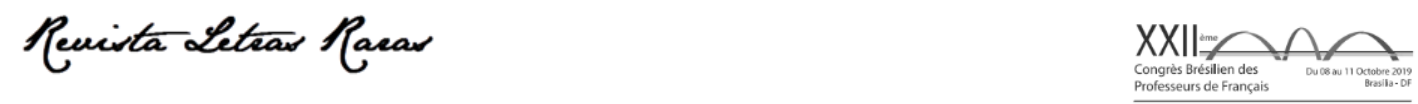

Actes du XXIlème Congrès brésilien des professeurs de français (dans Édition spéciale de la Revue Letras Raras 2020)

ISSN : 2317-2347 - v. 9, Dossier Spécial (2020)

Todo o conteúdo da RLR está licenciado sob Creative Commons Atribuição 4.0 Internacional

RÓNAI, P. Escola de tradutores. Rio de Janeiro: Nova Fronteira, 1976a.

RÓNAI, P. A tradução vivida. Rio de Janeiro: Educom, 1976b.

RÓNAI, P. Guia prático da tradução francesa. $3^{\text {a }}$ ed. Rio de Janeiro: Nova Fronteira, 1983.

SILVA, G. J. da; PONGE, R. Que signifie, comment traduire em portugais l'expression idiomatique "couper l'herbe sous le pied à quelqu'un»? In: XIV Congrès National des Professeurs de Français, 14, 2017, Mendoza (Argentina). Disponível em: [https://bdigital.uncu.edu.ar/fichas.php?idobjeto=9273>] Acesso em: 4 mai. 2020.

TLFi - TRÉSOR DE LA LANGUE FRANÇAISE INFORMATISÉ. Nancy: ATILF-CNRS/Université de Lorraine, 1994. Versão Online. Disponível em: [http://atilf.atilf.fr/]. Acesso em: 30 jun. 2020.

XATARA, C. M.; OLIVEIRA,W. A. L. de. Dicionário de falsos cognatos francês-português e português-francês. $2^{\mathrm{a}}$ ed. revista e ampliada. São Paulo: Editora de Cultura, 2008.

VINAY, J. P.; DARBELNET, J. Stylistique comparée du français et de l'anglais: méthode de traduction. Nouvelle édition revue et corrigée. Paris: Didier, 1972. 